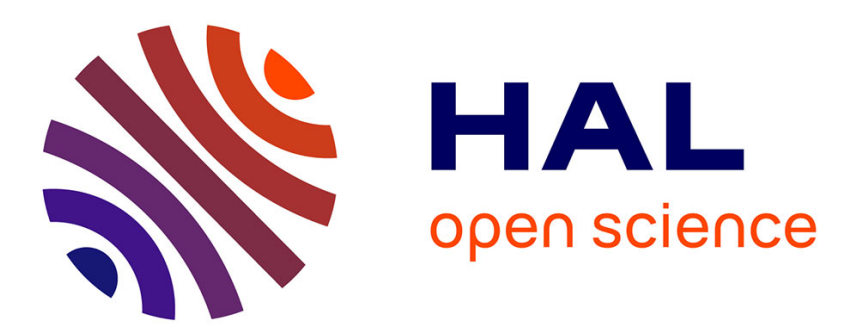

\title{
Vapor-liquid-solid mechanisms: Challenges for nanosized quantum cluster/dot/wire materials
}

Pierre Cheyssac, M. Sacilotti, G. Patriarche

\section{To cite this version:}

Pierre Cheyssac, M. Sacilotti, G. Patriarche. Vapor-liquid-solid mechanisms: Challenges for nanosized quantum cluster/dot/wire materials. Journal of Applied Physics, 2006, 100 (044315), pp.044315-1. $10.1063 / 1.2236163$. hal-00459474

\section{HAL Id: hal-00459474 \\ https://hal.science/hal-00459474}

Submitted on 24 Feb 2010

HAL is a multi-disciplinary open access archive for the deposit and dissemination of scientific research documents, whether they are published or not. The documents may come from teaching and research institutions in France or abroad, or from public or private research centers.
L'archive ouverte pluridisciplinaire HAL, est destinée au dépôt et à la diffusion de documents scientifiques de niveau recherche, publiés ou non, émanant des établissements d'enseignement et de recherche français ou étrangers, des laboratoires publics ou privés. 


\title{
Vapor-liquid-solid mechanisms: Challenges for nanosized quantum cluster/dot/wire materials
}

\author{
P. Cheyssac ${ }^{\text {a) }}$ \\ Laboratoire de Physique de la Matière Condensée, UMR6622-CNRS 6622, Université de Nice-Sophia \\ Antipolis, Parc Valrose, F-06108 Nice Cedex 2, France \\ M. Sacilotti \\ Groupe Couches Minces et Nanostructures, UFR Sciences et Techniques FR 2604, \\ Université de Bourgogne, 9 Avenue A. Savary, BP 47870, 21078 Dijon Cedex, France \\ G. Patriarche \\ Laboratoire de Photonique et de Nanostructures, UPR20-CNRS, Route de Nozay, \\ 91460 Marcoussis, France
}

(Received 15 June 2005; accepted 30 June 2006; published online 25 August 2006)

\begin{abstract}
The growth mechanism model of a nanoscaled material is a critical step that has to be refined for a better understanding of a nanostructure's dot/wire fabrication. To do so, the growth mechanism will be discussed in this paper and the influence of the size of the metallic nanocluster starting point, referred to later as "size effect," will be studied. Among many of the so-called size effects, a tremendous decrease of the melting point of the metallic nanocluster changes the physical properties as well as the physical/mechanical interactions inside the growing structure composed of a metallic dot on top of a column. The thermodynamic size effect is related to the bending or curvature of chains of atoms, giving rise to the weakening of bonds between them; this size or curvature effect is described and approached to crystal nanodot/wire growth. We will describe this effect as that of a "cooking machine" when the number of atoms decreases from $\sim 10^{23}$ at. $/ \mathrm{cm}^{3}$ for a bulk material to a few tens of them in a 1-2 nm diameter sphere. The decrease of the number of atoms in a metallic cluster from such an enormous quantity is accompanied by a lowering of the melting temperature that extends from 200 up to $1000 \mathrm{~K}$, depending on the metallic material and its size under study. In this respect, the vapor-liquid-solid (VLS) model, which is the most utilized growth mechanism for quantum nanowires and nanodots, is critically exposed to size or curvature effects (CEs). More precisely, interactions in the vicinity of the growth regions should be reexamined. Some results illustrating the growth of micrometer-/nanometer-sized materials are presented in order to corroborate the CE/VLS models utilized by many research groups in today's nanosciences world. Examples of metallic clusters and semiconducting wires will be presented. The results and comments presented in this paper can be seen as a challenge to be overcome. From them, we expect that in a near future an improved model can be exposed to the scientific community.
\end{abstract}

\section{INTRODUCTION}

Nanosized materials, of great interest for the future and more efficient everyday devices and machines, experience mechanical, electrical, and optical properties modified by quantum confinement. Their development requires an improvement of our knowledge on both growth techniques and growth mechanisms that take place when building nanosized dots and wire/tube shaped three-dimensional structures, referred hereafter as 3D-Ss, although it is more appropriate for balloons than for wires and quantum dots since they are closer to one-dimensional structures (1D-Ss) than wires. Among nanocolumn structures, one finds empty tubes such as carbon nanotubes and wires or rods. ${ }^{1,2}$ Many research groups utilize as catalysts metallic nanosized cluster materials (diameter $d$ from 1 to $50 \mathrm{~nm}$ ) as the "cooking machine" for building the whole structure of three-dimensional (3D)

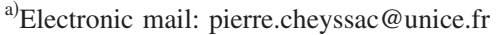

edifices, composed of a column topped by a nanosized cluster material. ${ }^{2-6}$ Others groups mention noncatalytic 3D-S growth. " 7,8 "Self-catalysts" have been also reported to grow 3D-S by using organometallic compounds that interact with many kinds of metallic, semiconductor, and insulating materials. ${ }^{9-11}$ One clearly sees that $3 \mathrm{D}$ shaped structures will be different according to the method of their fabrication, and that different applications can result from such diversities of structures.

Wire shaped materials have been realized since 1964, after Wagner and Ellis's work on the growth of micrometersized semiconductor materials. ${ }^{12}$ In their growth model, "food nutrients" are chemical elements that will be incorporated in the 3D-S through a catalysis effect due to the action of a "cooking agent," a gold metallic cluster. In other words, a micrometer-sized metal cluster acts as a catalyst, transforms food nutrients, and builds the 3D-S. The concept behind this model was a vapor-liquid-solid (VLS) mechanism in which the growth of 3D-S takes place by volume diffusion 
of transformed nutrients through the metallic catalyst cluster that builds the whole structure. In 1975, Givargizov's work proposed that the VLS model consisted of four steps, the more important being that which controls the metal cluster/ column growths at their interface. ${ }^{13}$ In this model, the growth environment can be physical vapor deposition (PVD) or chemical vapor deposition (CVD). ${ }^{12,13}$ More recently a chemistry research group, led by Buhro and co-workers, has shown that the VLS growth mechanism could be adapted to 3D-S growth in solution ambient and proposed the solutionliquid-solid (SLS) mechanism. ${ }^{14-16}$ In some of their work, Trentter et al. used nanosized decomposed metal-organic molecules for growing semiconductor materials at temperatures of 200-400 K below the current PVD or CVD process temperature. $^{14}$

In the race for more efficient building processes and the need for nanosized structures, the micrometallic cluster has reached the nanometer range. ${ }^{4,6,8,12,14}$ The physical and chemical properties of the cooking agent, the metallic cluster, are more or less modified by this reduction in size but many of the nanosized structure makers do not take such effects into account. Among these size effects, one finds catalytic properties whose efficiency may change with crystallographic faces of the metallic cluster, movement of its crystallographic planes and surface atoms, and the decrease of its melting temperature $T_{m}$ with size. ${ }^{17}$ Catalysis and diffusion are still parts of the previous growth models; their improvement and a better understanding of the existing experimental results should result if one takes such size effects into account. Wagner and Ellis ${ }^{12}$ and Givargizov, ${ }^{13}$ as well as more modern descriptions of growth methods such as metalorganic CVD (MOCVD), ${ }^{8}$ chemical beam epitaxy (CBE), ${ }^{5,6}$ laser catalytic growth (LCG) mechanism, ${ }^{4}$ and SLS, ${ }^{14-16}$ assume that the metallic cluster/column interface is flat; however, size effects or the physical state of the cluster itself (solid or liquid) may affect its curvature. The decrease of the melting temperature $T_{m}$ with the size of the metallic cluster has been studied by Buffat's work that applied the GibbsThomson equation to relate $T_{m}$ to the diameter of small and spherical metallic particles. ${ }^{17}$ Kofman and co-workers demonstrated that not only the diameter of the dot but also its shape, described by curvatures, modifies $T_{m}$. ${ }^{18,19}$ Then, the physical state and shape of the metallic cluster depend on its size. Such effects seem forgotten or not taken into account by many nanosized crystal growers.

The aim of this paper is to discuss the influence of metallic cluster size, shape, and state on the growth of 3D-S, related to some of our grown structures. Size effects involved in this paper are physical parameters such as $T_{m}$, crystallographic structure of the metallic cluster, mobility of its surface atoms, flatness of the cluster/column interface, diffusion of atoms (metallic cluster or column nutrients), and vapor pressure, due to the curvature of the metallic cluster surface. This paper will take into account most of the debated referenced works presented above; it will present the influence of size/shape effects upon the VLS mechanism and will propose some paths for research in the field of quantum dot and wire semiconductor materials to overcome the presently debated results. The flat cluster/column interface model by Wagner and Ellis and followed by most of cluster/column or tube growers is presently questioned. ${ }^{4-8,12-16}$ Inside the community debating the VLS/SLS models, recent results by Samuelson and co-workers led to the questioning of the diffusion through the metallic cluster and proposal of a more convenient surface reaction limited growth model. ${ }^{20,21}$ Melting temperature modified by the cluster size and shape, but not taken into account by most of modern wire growers, should also be taken into account. ${ }^{22,23}$ Throughout this paper and references therein the word column will refer to wire, rod, or tube and cluster to metallic aggregates used as the cooking system helping to build the 3D-S. This 3D-S will refer to a column and a metallic cluster on top of it.

This paper is organized as follows: (a) Some aspects of the VLS mechanism model and its drawbacks will be presented. (b) Shape/size effects will be exposed, and results about many metals catalysts presented. (c) Suggestions for a more convenient dot/rod interface model will be given, supported by some recent results about catalyst/noncatalyst 3D-S growth.

\section{THE MODEL OF THE VLS (SLS, VSS, ETC.) MECHANISM}

Demonstrated by Wagner and Ellis's work and followed by many other 3D-S growers such as Givargizov, Lieber, Samuelson, and Buhro, the Vapor-liquid-solid mechanism is briefly described below. ${ }^{2-16}$ Although this paper focuses on VLS and vapor-solid-solid (VSS) mechanisms, it is worthwhile to mention that there exist other possibilities. Carbon tubes have been grown without metallic cluster but with ferrocene as catalyst. ${ }^{24}$ Oxide assisted growth has also been performed. $^{25,26}$

For the first step of the VLS mechanism, a metallic layer or cluster is deposited at the surface of a flat substrate; with or without annealing, metallic aggregates or clusters form on this surface. Then, in this model, the flat interface that may exist between the cluster and the growing column comes from the existing one at the beginning of the process. In the second step, the surface is fed with nutrients in a PVD or CVD, assisted or not, environment. In liquid solutions, metallic particles can be added to the solution or created in situ by chemical reactions. ${ }^{14-16}$ According to this model, the nutrients cross the dot's metallic structure by diffusion and build an empty or filled column that is considered nowadays as a wire, nanowire, nanorod, or nanotube. The interface between the metallic cluster $(d \sim 10-50 \mathrm{~nm})$ and the column $\left(d_{c} \sim 10-50 \mathrm{~nm}\right)$ has always been considered as flat, ${ }^{2-6,12-16}$ the main reason for this being that $3 \mathrm{D}-\mathrm{S}$ results from an almost perfectly flat heteroepitaxy, ${ }^{20,21}$ as shown by highresolution transmission electron microscopy (HRTEM) pictures obtained after the 3D-S growth. In this respect, it would be very interesting to monitor the curvature of the interface during the growth process.

Figure 1 summarizes the existing VLS growth model, as depicted above. Figure 2 shows a truncated sphere (metallic cluster) on top of a column, separated by a flat interface. Three radii of curvature can be considered: $R_{N}$, the radius of a complete sphere, $d \sim 1-50 \mathrm{~nm} ; R_{\propto}$, the infinite radius of 


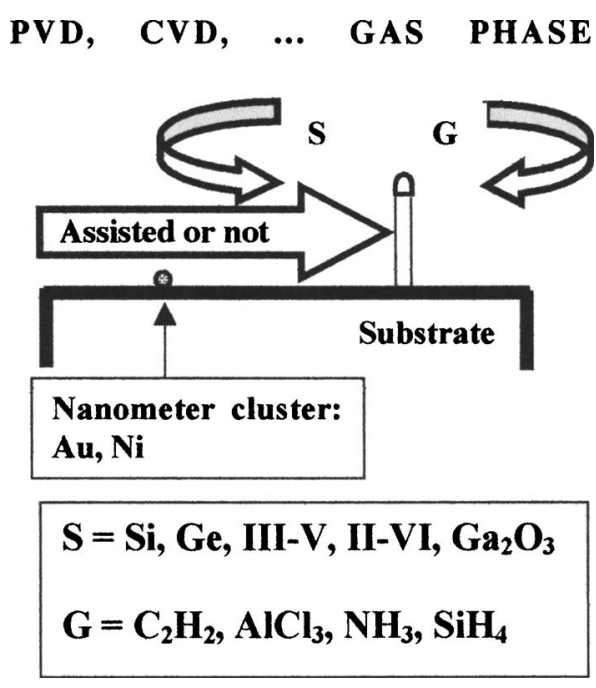

FIG. 1. A schematic representation model of the VLS mechanism: PVD or CVD growth of a nanotube/nanocolumn, starting from a metallic cluster and ending by a wire capped by a metallic cluster.

curvature of a flat interface between the cluster and the growing column [both are not supposed to be questioned since they represent, respectively, the sphere (a few tens of nanometers) and the radii of curvature of the cluster column assumed as a flat interface]; and $R_{c}$, the radius of the contact line or corner zone between the truncated metallic sphere and the flat interface. We leave apart the radius of the column that supports the interface.

Within the VLS model, the problem starts when considering $R_{c}$ : if it approaches zero or the interatomic distance, thermodynamic size or curvature effects should strongly apply. In the metallic truncated sphere, regions described essentially by $R_{c}$ present a melting temperature much lower than in the rest of the sphere. ${ }^{18,19}$ Let us take the example of a gold cluster whose bulk melting point is $T_{B} \sim 1336 \mathrm{~K}$; $T_{m}\left(R_{c} \sim 1.5 \mathrm{~nm}\right) \sim 765 \mathrm{~K}$ and $T_{m}$ reaches room temperature for $R_{c} \sim 0.8 \mathrm{~nm}$. Around $R_{N} \sim 2.5 \mathrm{~nm}, T_{m}$ changes very rap-

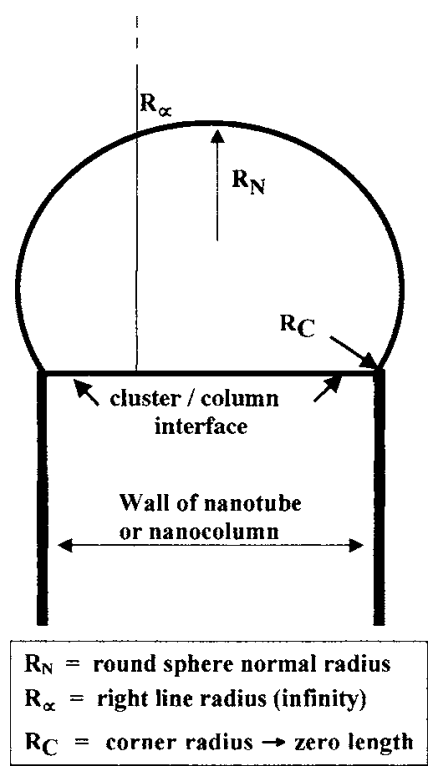

FIG. 2. The VLS mechanism; geometry of the existing metallic cluster/ column flat interface associated with the radii of curvature. idly. When $R_{N}$ goes from 10 to 2.5 and $1.65 \mathrm{~nm}, T_{m}\left(R_{N}\right.$ $\sim 10 \mathrm{~nm})=1261 \mathrm{~K}, T_{m}\left(R_{N} \sim 2.5 \mathrm{~nm}\right)=1080 \mathrm{~K}$, and $T_{m}\left(R_{N}\right.$ $\sim 1.65 \mathrm{~nm})=934 \mathrm{~K}$. One can evaluate the amplitude of these changes with $R_{N}$ through the relative variation of the melting temperature $\Delta T_{m} / T_{B}=\left(T_{B}-T_{m}\right) / T_{B}$ which takes the following values: $5.55 \times 10^{-2}, 19.1 \times 10^{-2}$, and $30.09 \times 10^{-2}$, respectively. ${ }^{17}$

If the VLS model is to remain with a cluster/column flat interface and a corner shaped gold cluster, its current state, then the corner contact line should be melted even at room temperature when $R_{c}<1.65 \mathrm{~nm} .{ }^{17}$ In this case, wetting conditions of the liquid part of the cluster onto the interface give rise to a contact angle that will determine the external shape of the gold cluster. This model should also present a local zone hotter than the others: the "cooking zone" is appropriated for building a columnar structure or a meniscus seeming like the one seen for bulk crystal growth from its liquid phase. ${ }^{27}$ Under these conditions, we enumerate below some points of the VLS model mechanism that may be criticized.

- The corner zone which is the external contact line that limits the flat interface between the truncated cluster and the column experiences a local decrease of the melting temperature of the supported metallic cluster due to curvature effects.

- A flat interface between the metallic cluster and the growing column is assumed once the growth process is running. Arguments are given below for other possibilities.

- Also, no physical reason justifies the existence of such flat interface between the metallic cluster and the mono- or multiwalled empty carbon column.

- More recently, Samuelson and co-workers have claimed a surface reaction mechanism instead of a volume diffusion limited reaction through the metallic cluster. $^{21,28}$

The first criticism has been examined above. Concerning the second one, in fact, physical requirements can be brought into play to clarify the assumption of a strictly flat clustercolumn interface. When two different materials, substrate and deposited cluster, are in contact, their common interface is an interaction zone, and each material exerts forces on the other one. These forces are interfacial tensions and determine the possibility of wetting of one material onto the other as well as the external shape of the contactless zones (Fig. 2). Wetting exists for solids as well as for liquids. If the substrate, i.e., in our case the column, is not rigid enough because of its thickness or its mechanical properties, its interface in contact with another material will be bent by the interfacial tensions exerted by this material. Then $R_{\propto}$ can no longer be considered as infinite. ${ }^{29-31}$ Also, the equilibrium between the various interfacial tensions governs the contact angle or wetting angle between the cluster and the column and also its external shape. ${ }^{32}$ This concept of wetting angle remains valid even for small sizes as considered here. ${ }^{33}$

The description of the cluster-column interface should then be improved in the following way: for most metallic cluster and growing column materials utilized, $R_{\propto}$ (Fig. 2) becomes finite and a characteristic contact angle is present 
between the metallic cluster (or crystal) and the column. Moreover, the previous external contact line should not remain as a circle on a flat interface with $R_{c} \sim 0$ but becomes a transition zone associated with $R_{c}$ larger than the interatomic distance of the cluster atoms.

During the very beginning of growth, if the substrate is flat, the cluster already on the substrate shares a flat interface with it before the column growth starts. Unless both cluster and column realize a perfect epitaxial contact at their interface, stresses are present due to interfacial energies and tend to bend this interface during the growth process. As a consequence, other phenomena should be invoked if one wants to keep a flat cluster-column interface during the growth procedure.

This debate can help in building a VLS growth mechanism physically current and acceptable. It will be developed below.

\section{SOME 3D STRUCTURES GROWN BY MOCVD WITH ORGANOMETALLIC MOLECULES ONLY}

MOCVD has been used since 1967 and crystal growers have since then discussed for many years metallic droplets that occur during high ratio growth conditions of III/V compounds. $^{34,35}$ The same effect holds for the molecular beam epitaxy (MBE) and other related techniques. ${ }^{36}$

Recently, 3D-Ss presenting top to bottom interfaces with other shapes have been grown by simply using the interaction of organometallic precursor molecules with substrates. These results are presented below, accompanied by information about size and curvature effects for the most commonly used metallic clusters.

Sacilotti et al. have shown that, if group $\mathrm{V}$ hydrides $\mathrm{H}^{+}$ are absent and when organometallic pyrolysis occurs in a MOCVD system, the deposit does not form metallic droplets lying on the substrate. ${ }^{9}$ A surprising $3 \mathrm{D}-\mathrm{S}$ is created during MOCVD with trimethyl gallium (TMGa). Gallium grows as top balls that are not in direct contact with the surface of the substrate but are attached to it via a cone shaped carbon base. ${ }^{9,10}$ Figure 3 presents some top and side views of these 3D composite structures. Scanning electron microscopy (SEM) coupled with energy dispersive $\mathrm{x}$-ray (EDX) analysis and transmission electron microscopy (TEM), respectively, have given their structure and composition. The cone shaped base is a very thin multiwalled membrane made of amorphous/turbostratic mixed carbon ${ }^{37-39}$ created after the $\left(\mathrm{CH}_{3}\right)_{3}$ decomposition/reaction of TMGa: ${ }^{40}$

$$
\begin{aligned}
& \mathrm{Ga}\left(\mathrm{CH}_{3}\right)_{3}(923 \mathrm{~K}) \\
& \quad \rightarrow \mathrm{Ga}_{\text {liquid }}+\cdots-\mathrm{C}-\mathrm{C}-\mathrm{C}-\mathrm{C}-\mathrm{C}-\mathrm{C}-\cdots \\
& \quad+x \mathrm{H}^{+} .
\end{aligned}
$$

If a preexisting metal lies on the substrate, the cone base may be taller. Examples of such 3D-Ss are shown in Figs. 3-5 which present balloons, nanowires, or scepters. They are not sensitive to gravity; they grow vertically on top of the substrate or horizontally on its sides, demonstrating the strength of the bonds between the substrate and the carbon base.

In Fig. 3(b), the substrate is bare, no metal acting as a catalyst being present as in the VLS model; the 3D structure

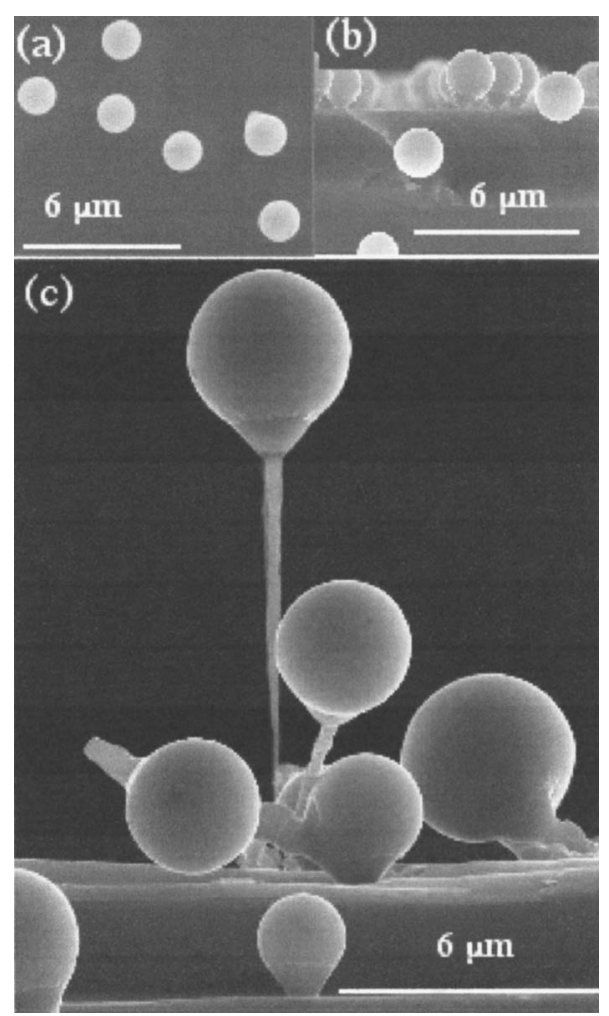

FIG. 3. MOCVD growth of $3 \mathrm{D}-\mathrm{S}$ at $650{ }^{\circ} \mathrm{C}$. (a) Top view of gallium spheres surrounded by a carbon envelope. (b) Side view of spheres lying on a carbon cone. The contrast allowed by SEM shows clearly the compositional difference between spheres and cones. (c) Scepter structure: a long and empty carbon structure capped by a Ga sphere covered with a thin $(10-25 \mathrm{~nm})$ amorphous carbon membrane. The substrate is (100) Si photolithographically prepared with $1-10 \mu \mathrm{m}$ width and $100 \mathrm{~nm}$ thick $\mathrm{Al}$ stripes.

looks like a montgolfier or balloon so (a) and (b) are called "montgolfiers." film/silicon; here also, no metallic cluster is acting as a catalyst; it shows scepterlike structures deposited by MOCVD on aluminum stripes. A carbon tube supports a much taller balloon. In the same way, $\mathrm{Cu}, \mathrm{Pt}, \mathrm{Au}$, or In may be used as substrates; 3D-Ss are built through their interaction with organometallic molecules.

Figures 4(a) and 4(b) depict nanowires made of $\mathrm{GaCuO}_{2}$, on $\sim 3.6 \mathrm{eV}$ band-gap semiconductor, created by the interaction of TMGa with a $\mathrm{Cu}$ substrate at $923 \mathrm{~K}^{41}$ As described in Ref. 41, oxygen comes from the surface of the copper oxide substrate.

Figure 4 and Ref. 41 show clearly that many types of curved or nearly flat interfaces exist between the metallic cluster and the column for the construction of these 3D-Ss. The same results hold for the GalnP bamboo shaped structure shown in Fig. 5, created by the interaction of TMGa with an InP substrate. ${ }^{11,42}$

Keeping in mind these results, we present below some important points about the curvature or size effect already presented in the literature but adapted here to nanocolumn aspects and most common catalyst metals utilized by most in the PVD and CVD crystal growers community.

\section{THE SIZE/SHAPE CLUSTER-COOKING MACHINE}

The bulk melting temperature $T_{B}$ of a material intervenes in various technological fields, in particular, metallurgy of 

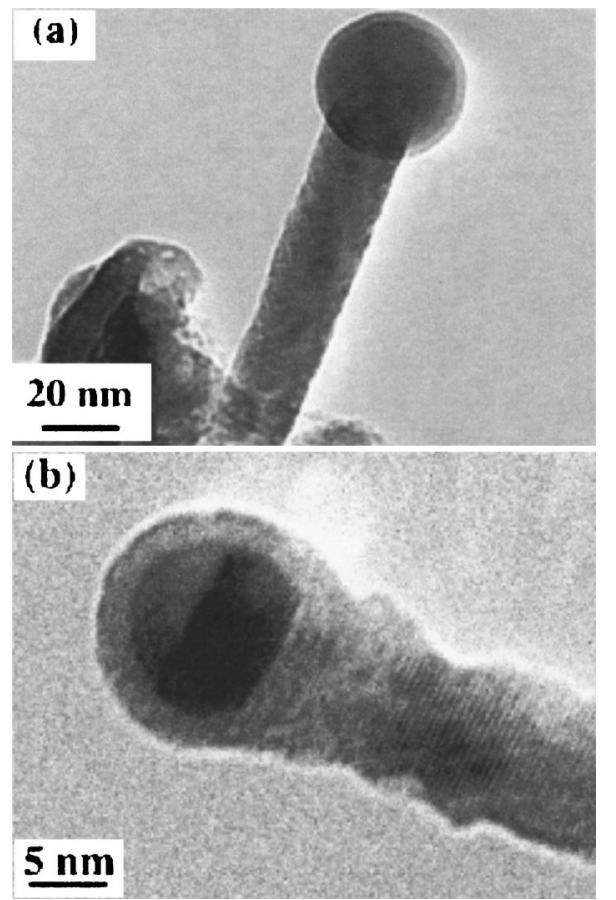

FIG. 4. $\mathrm{GaCuO}_{2}$ single nanowires grown on $\mathrm{Cu}$ substrates by MOCVD (Ref. 38) as seen by HRTEM: (a) rounded interface between the column and the cluster and (b) interstitial planes of a single crystal inside the column and bowed cluster/column interface.

most common metals and alloys such as gold, aluminum, iron, copper, stainless steel, etc. These materials are produced in large dimensions from millimeter to meter sizes and over but some are made of aggregated grains (micrometer size). When the grain size decreases down to the nanometer range, so does the melting temperature $T_{m}$ that noticeably deviates from $T_{B}$ (Refs. 17-19) beginning at a few micrometers. This phenomenon is not widely known; many but not all of its aspects are now understood; apart from sintering, we are not aware of applications to modern material production. Modern microelectronic and optoelectronic industries use ultrapure metals and their alloys: $\mathrm{Au}, \mathrm{Al}, \mathrm{In}, \mathrm{W}, \mathrm{Ti}, \mathrm{Ni}, \mathrm{Ag}, \mathrm{Cu}$, $\mathrm{Zn}, \mathrm{Pt}, \mathrm{Nb}$, etc. The use of metals of high purity and of nanosized dimensions open insights into other properties. When a cluster, aggregate of atoms, is a few nanometers in size, size effect appears as a phenomenon associated with curvature effect, and atoms do not behave anymore as in bulk material. This curvature effect leads to the bending of chains of atoms and a decrease of the strength of atom bond, as depicted by Fig. 6, that presents two bent chains of atoms

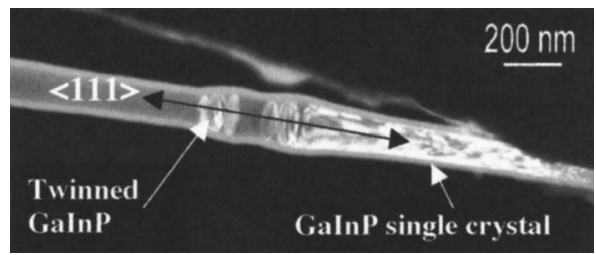

FIG. 5. HRTEM picture of a GaInP nanowire obtained by MOCVD, created by the interaction of TMGa with an InP substrate held at $650^{\circ} \mathrm{C}$ (Ref. 39). The nanowire rod region is composed of GaInP disks rotated by $60^{\circ}$ from each other.

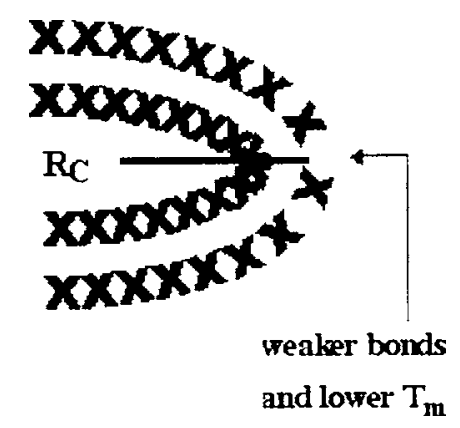

FIG. 6. Two bent chains of atoms showing schematically that surface atoms are more weakly bound to their neighbors than the inner ones. $R_{c}$ is the corner radius, decreased by the bending.

to exemplify this effect. Experimental results have shown that surface atoms of a cluster are more exposed to this curvature effect.

Melting comes from the influence of surface atoms on the volume. In other words, there is a competition between Gibbs free energies of surface and inner atoms of the cluster. Semenchenko's model is among the simplest giving a variation of the melting point with size. Input parameters are the latent heat $L$ of melting, the diameter $d$ of the metallic spherical particle, its bulk melting point $T_{B}$, the interfacial tension $\gamma_{\text {ls }}$ between the solid and the liquid phase of the material under study, and the density $\rho$. It gives $T_{m}(d)$ $=T_{B} \exp \left(-4 \gamma_{\mathrm{sl}} / \rho L d\right) .{ }^{43}$

More elaborated models, which differ by the description and interpretation of melting, have been devised but, whatever they are, at first order, $T_{m}(d) / T_{B} \sim 1-A / d$, where $A$ encloses $\gamma_{\mathrm{Is}} \rho$ and $L .^{17,44}$ The numerical values obtained with various models are relatively close; so Semenchenko's relation is sufficient. When clusters have a low number of atoms, A/d approaches 1 and melting is not very clearly defined. Solid-liquid phase fluctuations occur ${ }^{45,46}$ and $T_{m}$ is no longer a continuous function of the number of atoms. ${ }^{47}$

As an example, a high $T_{m}$ material such as gold melts at $1336 \mathrm{~K}$ and $T_{m}(d)$ follows the behavior shown in Ref. 17. One can see that a $1 \mathrm{~nm}$ diameter spherical Au cluster contains $\sim 25$ atom melts below $300 \mathrm{~K}$. Bulk gallium which is the $\alpha$ phase of Ga, a low $T_{m}$ material, melts at $302.9 \mathrm{~K}$. Once divided into small droplets, $\mathrm{Ga}$ is quite special because it no longer exhibits its bulk $\alpha$ phase but other crystallographic phases, $\beta, \gamma, \delta$, and $\varepsilon$ with different melting temperatures, for example, $T_{B} \quad(\mathrm{Ga} \beta)=256.85 \mathrm{~K}$ and $T_{B} \quad(\mathrm{Ga} \delta)$ $=253.75 \mathrm{~K}$. Figure 7 shows the behavior of the $\mathrm{Ga} \beta$ and $\mathrm{Ga}$ $\delta$ phases as a function of $d$. A $1 \mathrm{~nm}$ diameter spherical cluster contains $\sim 31$ atoms and melts below $170 \mathrm{~K}$. The melting temperatures $T_{m}(d)$ of high $T_{B}$ materials such as $\mathrm{Fe}$ and $\mathrm{Ni}$ follow the same behavior as that of Au described in Ref. 17. This demonstrates that the size of the top cluster may influence its state, solid or liquid, according to the temperature of the growth process and so is able to modify the 3D-S growth conditions.

Melting begins at places on the surface where the local radius of curvature is the smallest; a solid-liquid equilibrium exists over a few degrees before the end of melting being reached. ${ }^{23}$ Curvature effects exist for rounded or spherical surfaces; they obviously exist also for other shapes such as 


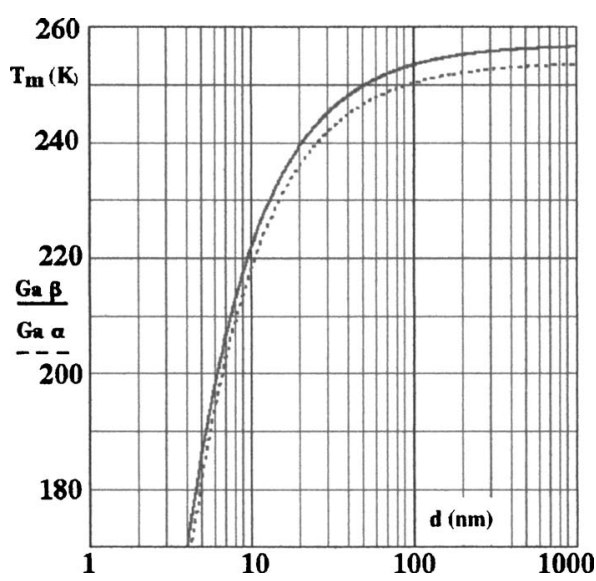

FIG. 7. Melting temperature $T_{m}$ of Ga $\beta$ and Ga $\delta$ clusters as a function of their diameter $d: T_{B} \mathrm{Ga} \beta=256.85 \mathrm{~K}$ and $T_{B} \mathrm{Ga} \delta=253.75 \mathrm{~K}$.

grain corners or edges. ${ }^{48,49}$ Figures 8 and 9 show dark field TEM pictures of $\mathrm{Pb}$ clusters around $561 \mathrm{~K}\left(T_{B}=600.56 \mathrm{~K}\right)^{18}$ and $\mathrm{Ga}$ at $250 \mathrm{~K}$ for small size and deformed shape clusters, respectively. ${ }^{19,50}$

Note that, in Figs. 8 and 9, a big and a small sphere that coalesce create a nonspherical particle, for which melting begins at places where the radius of curvature is the smallest and at a temperature lower than that of the bigger sphere. Nature has gifted us with this phenomenon even for micrometer-size metallic growth. Facing these arguments and facts with the present science of nano- and microcrystal growth, we should be able to introduce different concepts. These concepts will be mentioned for atom by atom growth, growth of dots by solidifying melted metallic clusters, and nanocolumn growth.

\section{IMPROVEMENT OF THE VLS GROWTH MODEL}

We have shown that the VLS model in its present state is to be questioned and a more refined explanation should be found if one wants to explain the unusual structures exhibited by Fig. 3 and particularly the 3D scepters [Figs. 3(b) and $3(\mathrm{c})]$.

Let us recall the geometric description as shown by the various samples. Seen from the top, the 3D-Ss look like spheres [Fig. 3(a)]; the side view shows that they are actually made of spheres supported by a cone, whose area in contact with the substrate is fairly small, diameter less than $10 \mathrm{~nm}$. The top ball is a sphere to a very good accuracy. Its diameter parallel to the substrate is $\sim 2 \mu \mathrm{m}$ while the top to bottom distance is $\sim 2.35 \mu \mathrm{m}$ [Fig. 3(b)]. The height of the cone is $\sim 0.428 \mu \mathrm{m}$; its diameter measured at the interface between the bottom of the cone and the ball is $\sim 1 \mu \mathrm{m}$; this corresponds to $\mathrm{a} \sim 45^{\circ}$ half-angle at the summit. The ball and its associated supporting cone are attached to the substrate by an empty column (a carbon tube) whose diameter is at least one order of magnitude less that the ball's diameter [Fig. 3(c)]. For such scepter shown in Fig. 3(c), the diameters of the ball and the column (tube) are 3.69 and $0.13 \mu \mathrm{m}$, respectively; the column length is $9.40 \mu \mathrm{m}$. The half-angle of the cone is $\sim 40^{\circ}$ and the ball-cone contact angle is $\sim 138^{\circ}$. At the beginning of the growth process, the growing sphere presents a
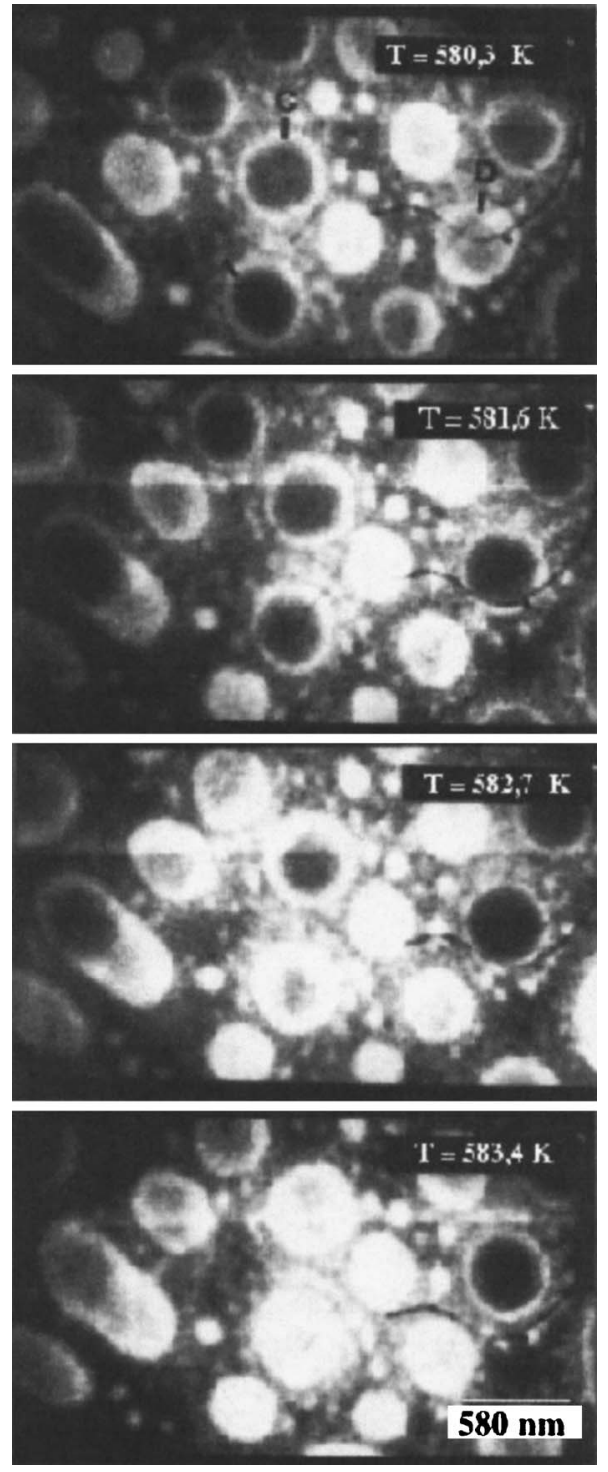

FIG. 8. Dark field transmission electron microscopy (DFTEM) of spherical and nonspherical lead particles embedded in a $\mathrm{SiO}$ matrix, $T_{B}=600.5 \mathrm{~K}$, taken from Ref. 23. Black areas C and D are associated with solid parts, bright ones are associated with solid parts in Bragg conditions for the electron beam, and areas of intermediate gray levels represent electrons scattered incoherently by the liquid parts of the particles.

flat interface with the thick and flat substrate. During the column growth, this interface (ball/column) may no longer remain flat as it appears in Fig. 4.

Chemical and structural analyses of these 3D-Ss, performed using SEM, EDX, and TEM, have shown that the ball is mainly composed of liquid gallium, the cone being made of carbon for which more details are found in Refs. 10 and 51. If the ball-cone interface was to be flat, the $\mathrm{Ga}-\mathrm{C}$ contact angle calculated from the geometry of the system would be $\sim 125^{\circ}$. This is in good agreement with previous measurements of wetting angles of very high purity $\mathrm{Ga}$ on clean $\mathrm{Al}_{2} \mathrm{O}_{3}$ single crystal; $\mathrm{Ga}$ exhibits a contact angle in the range of $118^{\circ}-130^{\circ}$, that usually decreases if few metallic impurities are present on the substrate. ${ }^{52,53}$

The questions are as follows: How does the growth process start? What can be the driving forces (catalysis, diffu- 


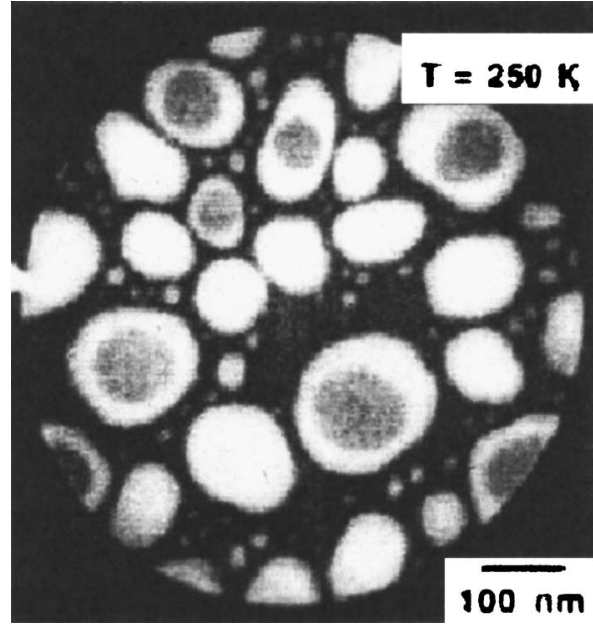

FIG. 9. DFTEM of spherical and nonspherical gallium particles embedded in $\mathrm{a} \mathrm{SiO}_{x}$ matrix, taken from Ref. 50 .

sion, segregation, etc.) building the whole $3 \mathrm{D}-\mathrm{S}$ shown in Fig. 3 (ball, cone, and tube)? They are discussed in what follows.

\section{HOW DOES THE GROWTH PROCESS START?}

Generally, one can distinguish between Volmer-Weber ${ }^{54}$ and Frank-van der Merve ${ }^{55}$ growth modes; in between is Stransky-Krastanov's mode, a mixture of both the previous ones; one atom is the elemental brick. The way these atoms realize three-dimensional clusters, islands, or continuous films, amorphous or crystalline structures, depends on parameters related to the material under growth, its substrate, and the experimental conditions of the growth itself. Experimental conditions such as patterning etching, chemical synthesis, stress driven organization, growth temperature, etc., are different. In what follows, but not relevant on the process itself, we examine how the presence or absence of a metallic cluster with its associated size effect may affect growth processes.

\section{A. Atom by atom crystal growth}

Currently, most of nanosized single crystal growers utilize PVD and CVD methods such as $\mathrm{MBE},{ }^{33} \mathrm{CBE},{ }^{5,6}$ $\mathrm{LCG}^{2-4}$ MOCVD ${ }^{8-11,34}$ metal-organic vapor phase epitaxy (MOVPE), ${ }^{35}$ and atomic layer epitaxy (ALE) or atomic layer deposition (ALD). ${ }^{56}$ In these various methods, a spatially continuous film crystal growth reduces to an atom by atom building process of its structure; atoms or molecules reach the substrate as individuals or partly cracked entities, organize themselves, and form the crystal layer by layer. During the first steps (a few atoms) of the formation of a growing cluster, temperature plays a role. Due to its size, the growing cluster is deeply into the conditions presented in Figs. 7-9; it experiences the thermodynamic size effect and its growth takes place in the liquid state. For most of PVD/CVD methods, growth nutrients are in the condition where the vaporliquid-solid mechanism holds, As an example, MOCVD or MBE growth of GaAs. ${ }^{34-36}$ Cracking of nutrients for epilayer growth needs relatively high temperatures $(800$
$-1000 \mathrm{~K}) ;^{7-10,35}$ on the contrary, thermal requirements are much lower in liquid solutions, down to $400 \mathrm{~K}$ and below, and VLS or SLS mechanisms hold in the same way. ${ }^{14-16}$ Achievement of lower thermal requirements is then obtained using a small liquid metallic cluster whose melting temperature has been lowered, thanks to the size effect. Of course, as size increases and reaches the micrometer range, the influence of the thermodynamic size effect on the metallic cluster, and so on the growth conditions, decreases strongly and disappears.

\section{B. Nanocolumn growth assisted by a metallic cluster}

We have shown in Sec. II that the VLS growth mechanism suffers actually from unresolved questions that can find their origin in size effects as described above. The VLS model, accepted as it is, imposes a metallic ring, limiting a meniscus, on top of the growing column and between the flat-rounded regions. The radius of curvature of this ring is in the range where size effects cannot be neglected. Moreover, catalysis takes place on the whole surface of the metallic cluster, a process that releases heat. In the vicinity of the ring, exothermic catalysis should be more active than elsewhere because species cracked there are used for building the column; then more heat should be released close to the ring; its temperature is higher than in other places on the metallic cluster surface and the rest of the column. The temperature of the ring is then higher than the melting one of, for example, if used, a $10 \mathrm{~nm}$ diameter gold cluster. ${ }^{17}$ A gold cluster may exhibit solid zones associated with low curvatures and liquid zones associated with high curvatures for which the size effect applies.

How can one reconcile rounded and flat cluster/column interfaces? At the beginning of the growth process, the cluster lies on the substrate and the contact zone reproduces its curvature, in our case a flat interface. As soon as growth begins in the region located between the cluster and the substrate, interfacial tensions appear and the associated forces determine the external shape of the cluster as well as the curvature of the interface. The cluster remains on top of the growing column and their interface may be bended, depending on the various forces that are exerted on both materials on each side. Then, if a flat cluster/column interface is to appear after the growth, it must be due to the subsequent step related to the column formation or evolution. Arguments based on these facts do not evacuate the physical problem. Now, surface tension and interfacial tensions that govern, respectively, the shape of the metallic cluster and the convexity of the cluster/column interface may be not strong enough to create a visible bending. Unfortunately, HRTEM pictures are obtained after growth has stopped; relaxation processes may have taken place leading to flat interfaces. This does not prevent curved interfaces from existing: the visible curvature is created by couples of materials in contact, such as cluster/growing column, for which the ratio of interfacial forces to Young modulus enables such a visible bending to occur. This may explain why various convexities of cluster/growing column interfaces are found, ${ }^{41}$ depending on such couples of materials (Fig. 4). 
In this way, the VLS model by Wagner and Ellis still holds, although it has to be modified taking into account the following points.

(a) A metallic cluster and its surface atoms exhibit, respectively, curvature effects and diffusion process.

(b) The different materials build the column following the general lines described above: curvature effects added to diffusion processes, etc.

(c) The surface of the cluster plays a twofold role: catalysis and surface diffusion of cracked species toward the growing zone. The last point, diffusion, is the most important step and is described in more detail below. Mainly, the growth mechanism by diffusion of nutrients through the cluster has to be replaced by a surface controlled growth mechanism, as recently proposed in the case of nanowires and nanotrees. ${ }^{28}$

Suggestions and arguments for a more acceptable model are developed in the next paragraphs.

\section{Arguments for a surface diffusion model}

We have seen that a more convenient model of cluster/ column growth has to be set up. The metallic cluster, the cooking machine, acts as a catalyst; its surface or part of it may be in the solid or liquid state. ${ }^{17}$ The nutrients cracked at their surface are supposed to travel through the cluster toward the growing zone, namely, the cluster/column interface, and create the 3D-S (Fig. 2). ${ }^{12}$ We bring arguments in favor of surface diffusion as proposed in Ref. 28.

The temperature of the growth process and the small size of the metallic cluster act in the same direction: the melting temperature of curved zones decreases and the smaller the curvature, the bigger this decrease ${ }^{17}$ Near the contact zone of the cluster on the growing column, the cluster exhibits the highest curvature; this ring may be liquid while the rest of the cluster is in the solid state. However, for the sake of clarity, we will not go into such details and will keep a given state for the cluster.

If solid, most of the time, small metallic clusters are single crystals. Gold particles exhibit compact (111) surfaces whose surface tension is minimum; they appear as practically spherical under TEM for sizes lower than $10 \mathrm{~nm}$ diameter. ${ }^{17}$ High-resolution electron microscopy of $\mathrm{Pb}$ particles reveals the existence of facets and also rapid changes of the orientation of the crystallographic structure, even at temperatures lower than $T_{m}$. Although the cluster does not rotate as a whole, the orientations of its atomic planes are changing all the time, as demonstrated in Ref. 50. In such a case, it is difficult to understand how atoms diffuse through an evolving crystalline structure and reach the growing interface. As a matter of fact, the atoms of nutrients that build the column and have reacted at the surface of the cluster would have to channel through its moving atomic planes and keep the direction of the growing interface without having any driving force to go to the considered interface (cluster/ column). Whatever the diffusion model used (interstitial, substitution, etc.), the atoms diffusing inside the cluster will be guided by channeling processes and follow their initial diffusion direction; it is very unlikely that this direction coincides with that of the cluster/column interface.

If the cluster is liquid, crystallographic planes are smeared out and atoms are subjected to Brownian motion; the arguments against diffusion through the volume remain valid. Interactions of a given species of atoms with those of the cluster do change at its melting; however, this change is not so abrupt compared to that of other physical parameters such as electrical resistivity. Above melting, the surface is "more liquid" than the volume (Figs. 8 and 9). Simulations show that atoms move on the surface more than inside where the interaction with others remains uniform as an average, not giving rise to a driving force. ${ }^{57}$

Previous high-resolution electron microscopy experiments have shown that a gold single crystal experiences evaporation condensation at the atomic scale as well as diffusion of its surface atoms; ${ }^{58-60}$ coalescence between crystalline clusters also occurs from surface atoms and not from volume ones. ${ }^{61}$ In this context, it is more likely that nutrient atoms cracked at the metallic cluster surface behave in the same manner: they diffuse along the outer surface of the cluster instead of penetrating it. This is to be related with a nowadays generally accepted view that, in MOVPE, decomposition of precursor materials happens to a large degree outside the cluster/wire structure. The vicinity of the surface cluster may play both roles: catalyst and collector of decomposition products.

So, whatever the states of the catalyst cluster are, what can be the origin of a force driving the nutrients to the growing zone?

Let us show that this force is a consequence of the diffusion of nutrient atoms along the surface of the catalyst cluster and of the beginning of the growth process. Before growth starts, surface atoms of the metallic cluster itself are able to move on the surface, activated by thermal energy. Surface energy tends to a minimum and so, for a given number of atoms, the free surface of the cluster takes roughly a spherical shape except at the contact zone with the substrate where it imposes its own shape. When nutrients are created at the surface, they experience Brownian motion and show instantaneous velocities with random directions but there is still an intrinsic probability that they establish chemical bonds. The existence of the ring, contact line between the cluster and its substrate, modifies the picture: part of the random character of velocities is lost. They must reverse their orientations and cross a null value, and then nutrient atoms that were neighbors when entering this zone spend more time close together than in other zones on the cluster surface. The probability that they establish bonds is increased there and will remain always higher than elsewhere: the growth process from nutrient atoms starts from the contact ring.

Once growth has started, it consumes nutrient atoms; the growing zone (the cluster/column interface) exhibits a lower concentration of free nutrients than the rest of the cluster surface. Then, a gradient of concentration exists along the cluster surface. Classically, a driving force is associated with this gradient and creates a flux of nutrients toward the ring and the growing zone, the metallic cluster/column interface. 
Returning to the solid or liquid state of the catalyst cluster, the liquid cluster exhibits a truncated sphere shape lying on the interface with the growing column. Catalysis occurs on its surface; the diffusion coefficient of atoms on the surface of a liquid is higher than on or inside a solid. The gradient of nutrient concentration still exists but is higher for a liquid cluster than for a solid one, leading to a higher growth rate and efficiency of the process. If the catalyst cluster exhibits solid and liquid zones, the previous arguments remain valid.

Another argument for a surface diffusion assisted growth comes again from wetting or interfacial forces. When one considers a mixture of two different metals, at a temperature such as both are in the liquid state, it may exist as a domain of miscibility and wetting. Above some critical temperature, the metal with the lower interfacial energy segregates at the outside of the other one. ${ }^{62,63}$ Such a segregation strongly reduces the probability of finding atoms of the segregated metal below the few layers confined close to the exterior. It is possible to propose a volume diffusion of $\mathrm{C}$ inside the liquid Ga cluster toward the interface? It requires that some miscibility domain or compound between carbon and gallium exist. We are not aware of any $\mathrm{C}-\mathrm{Ga}$ phase diagram nor $\mathrm{GaC}$ carbide. Experiments done on carbon nanotubes filled by $\mathrm{Ga}$ and exposed to temperature higher than $700 \mathrm{~K}$ do not show the formation of a $\mathrm{Ga}-\mathrm{C}$ compound. ${ }^{64,65}$ Moreover, in the experiments reported here, because of the low temperature, and although $\mathrm{C}$ atoms are not equivalent to metallic ones, they cannot be considered as in a "liquid state." As a consequence, $\mathrm{C}$ should not penetrate the liquid metallic cluster over a few layers; a dewetting should occur between liquid Ga and "solid" carbon, favoring a surface diffusion process. Once a thin layer of $\mathrm{C}$ atoms covers a $\mathrm{Ga}$ droplet, although temperature is not comparable, the experimental situation is similar to that of liquid Ga in contact with diamond. In this case, it has been shown that the liquid layers of $\mathrm{Ga}$ in close contact with diamond experience an ordering and assume a solidlike structure that should influence its heterogeneous nucleation. ${ }^{66}$ Then, at low temperature, Ga should nucleate and freeze. In our case (Figs. 3-5), temperature is higher than the nucleation temperature of solid $\mathrm{Ga}$ and $\mathrm{Ga}$ remains liquid, but nothing prevents $\mathrm{C}$ atoms on the surface from organizing themselves, ${ }^{67}$ helped by the underlying organization of liquid Ga.

As a summary, temperature controls the solid or liquid state of the cluster, its chemical activity as a catalyst, the amplitude of characteristic parameters of surface diffusion, and the intrinsic probability of forming bonds. Surface diffusion controls both the external shape of the catalyst cluster even in the solid state and the growth process. The presence of the contact ring between the cluster and its substrate increases the probability of forming chemical bonds close to it; the concentration gradient of free nutrients as created gives rise to the driving force that leads atoms in the growing zone, the cluster/column interface.

\section{MOCVD growth of 3D-S perpendicular to the substrate}

As shown in Fig. 3(b), 3D-Ss grow up perpendicularly to the surface of single crystal or amorphous flat substrates. $^{9-11,40}$ There is no relation of heteroepitaxy between the crystalline state of the substrate and the 3D scepter structure composed of a carbon cone shaped base and a metallic sphere on top: the 3D-S does not copy the underlying crystallographic states. ${ }^{51}$ This is not always the case; it may happen that a metallic epitaxial growth reproduces the underlying structure of a crystalline substrate although separated from it by an amorphous and dielectric layer a few nanometer thick. ${ }^{66}$ However, even in this case, the epitaxy takes place perpendicularly to the substrate.

Defects, impurities, and steps are present on the substrate and are randomly distributed. During the MOCVD process, atoms diffuse on and above the substrate and are anchored by these defects/impurities and then a preferential growth of the catalyst cluster takes place at these points. The first step of growth occurs in the liquid state, because of the thermodynamic size effect, helped by the heating of the substrate; wetting conditions determine the characteristic shape of the truncated sphere of the clusters as demonstrated in Fig. 3 .

A cone shaped carbon base, or carbon column, has been evidenced for these 3D-Ss; the first 3D-S seed comes very likely from a surface interaction between a carbon radical and the substrate. ${ }^{51}$ The TMGa molecule gives the carbon radical. The carbon base and sphere grow together. The contact zone between the conical carbon base and the gallium cluster can be seen as the cooking machine described above. The beginning of the seed formation is facilitated by the existence of the first $\mathrm{Ga}-\mathrm{C}$ atoms that strongly experience the size effect because of the low content of Ga atoms. During the MOCVD process, Ga and carbon keep being fed to both cluster and cone, then the cone and the carbon membrane that surrounds the cluster have to grow or expand.

It is known that in comparable experimental situations, carbon nanotubes grow perpendicularly to a $\mathrm{Si}(001) / \mathrm{SiO}_{2}$ substrate ${ }^{68}$ the growth mechanism is helped by the presence of metallic clusters that act as catalysts. The growth process of the column may start from the contact zone or ring: it forms a carbon cone and a metallic cluster detaches from the substrate. If no more nutrients are injected, nothing changes. If nutrients are injected by the CVD phase or from the substrate itself, then the carbon cone expands in a column [Figs. 3(b) and 3(c)] from the ring, perpendicularly to the substrate. This is supported by experiments on the growth of multiwalled carbon nanotubes from a metallic cluster, for which a proposed mechanism among others would be stresses. ${ }^{69}$ Then, two possibilities have to be considered: either the cluster remains on the substrate and the carbon column grows from the cluster and in the opposite direction to the substrate, ${ }^{69}$ or the cluster is detached from the substrate. This last possibility is exemplified by multiwalled nanotubes that grow from a metallic Ni layer deposited onto $\mathrm{Si}(001) / \mathrm{SiO}_{2}$. $\mathrm{Ni}$ is detached from the substrate during the growth 


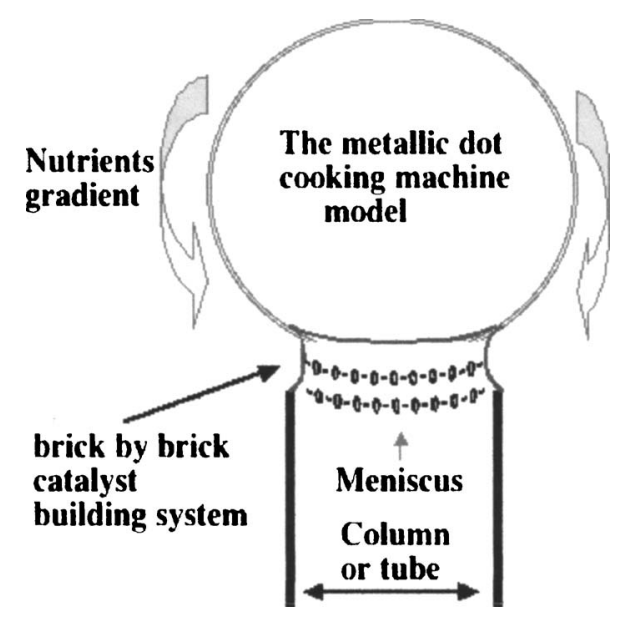

FIG. 10. Model of the construction of a column by a 3D-S metallic dot cooking machine: the sphere/column interface.

process. $^{70}$ Then, the column may grow in between the substrate and the metallic cluster (Figs. 4 and 5).

In the liquid state, more than in the solid state, the cluster/substrate catalyst couple exhibits an axis of symmetry, perpendicular to the substrate, and it remains unchanged under a rotation around it. At the beginning of the growth process, the probability that bonds form along the carbon cone/cluster interface is also isotropic compared to this axis. As a consequence, the $3 \mathrm{D}-\mathrm{S}$ should grow perpendicular to the substrate and should keep this symmetry. This is what Fig. 3(b) shows. However, it is known that directions of growth other than perpendicular to the substrate can be realized and their origin may be found in some broken symmetry, surface steps, and surface exposed alloys [see some 3D-Ss in Figs. 3(b) and 3(c)].

When metallic films are present on the substrate, they create preferential sites of nucleation for the catalyst clusters; they also constitute sources of atoms for alloying and rotational symmetry of the growth conditions may be broken. The direction of growth remains no longer perpendicular to the substrate and more complicated 3D-Ss can be obtained in these conditions [see Figs. 3(c), 4, and 5]. ${ }^{9-11,40-42}$

Keeping in mind the previous arguments and the experimental results that support them, we propose to improve the so-called VLS growth model according to a scheme described in Fig. 10.

A metallic cluster, or cooking machine, acts as a catalyst that feeds nutrients. Its surface or parts of it may be in the liquid state, due to the thermodynamic size effect added to the heat released from catalysis and heated substrate. Surface diffusion of nutrients helps building brick by brick (atoms) the column. Minimization of the various surface energies governs the convexities of the various interfaces, cluster/ column and column/substrate; structural relations govern the structure of the growing column. An argument of this scheme uses the decrease of the melting temperature of a localized zone in the vicinity of the cluster/column interface, a zone which is responsible for the brick by brick construction of the structure.

The brick by brick construction of the single or multiwalled carbon tube can be regarded in the same model: the physical state of the metallic cluster (entirely liquid or some part of its surface liquid and some solid) gives rise to the skeleton to be produced.

\section{How to choose the metallic catalyst cluster}

Although the answer to this question does not modify what has been presented before, such a choice governs the chemical reaction to be favored and the efficiency of the catalytic process. As an example, gold is preferred for the growth of carbon nanotubes and III-V compounds. ${ }^{2-8}$ Such a choice presents many advantages: a very weak affinity to oxygen, a low vapor pressure up to $T_{m}$ (no evaporation or an extremely low one) during the experiment, and the remaining constant of the size of the cluster. In such a respect, Ga also presents a very low vapor pressure, remains liquid over an extended temperature range, and its cluster keeps a constant shape of truncated sphere whatever the conventional growth temperature is.

It shows that the nature of atoms is involved but not their physical state (liquid or solid). In this respect one can understand that the physical state (liquid or solid) of the metallic catalyst cluster is not so important for the catalysis process.

Let us examine the influence of the size of the catalyst cluster. Let $N_{S}$ and $N_{V}$ be, respectively, the number of atoms located on the surface and the whole number of atoms in the cluster. Bulk material is characterized by $N_{S} / N_{V} \approx 0$, while it is 1 for a single atom. A decrease of size is accompanied by an increase of $N_{S} / N_{V}$. A spherical cluster of diameter $d$ and a cubic crystallographic structure with a lattice parameter $b$ give $N_{S} / N_{V}=3 b / d$; for example, a $d=3 \mathrm{~mm}$ lead cluster gives $N_{S} / N_{V} \sim 0.5$. Gold has the same crystallographic structure as lead, although its lattice parameter is slightly different; its $N_{S} / N_{V}$ remains quite comparable to 0.5 . Most of atoms from very small clusters are located at its surface, increasing the efficiency of the catalytic process.

\section{CONCLUSION}

The growth of modern materials structures with three dimensions (nanowires, columns, balloons, scepters) has been presented and discussed in relation to the melting temperature of nanosized metallic cluster acting as catalysts, which we call the cooking machine. The VLS growth mechanism proposed in the 1960s has been discussed and approached to more recent growth descriptions of 3D-S materials. Organometallic self-catalysts used for the construction of 3D-S materials have been incorporated into today's information and literature on existing 3D-S. The currently neglected melting temperature of nanosized metallic materials has been applied to the known growth of 3D shape columnar materials. The melting temperature decreases with the size of the metallic particle. The shape of nanosized materials (as a consequence of the surface curvature effect) has also been related to existing growth in nanomaterials science. It seems that a surface driven 3D-S growth mechanism would be more appropriate than the initially proposed diffusion mechanism through the volume and would give a better insight into an actual VLS mechanism of 3D-S growth. The metallic cluster-cooking machine that has been presented, 
discussed and extended to many existing growth techniques, can help in a future and more efficient description of a complete physical micro-/nanosized column growth mechanism. The size and shape of metallic cluster can be responsible for many and intriguing results related to $3 \mathrm{D}-\mathrm{S}$ growth. Some aspects of many and not yet physically related facts such as twining and/or kinking through the growth direction of wires, single/multiwalled carbon tubes, cluster/wire flatness or curvature of interfaces, and tapering have been examined through size effects that a metallic cluster experiences. A few recent results about MOCVD, assisted growth of 3D-S organometallic self-catalysts, have been presented and deal with the growth of metallic/carbon and metallic/semiconducting structures. GaInP and $\mathrm{GaCuO}_{2}$ nanosized wires as well as micrometer-sized GaN crystals have been obtained by the organometallic precursor interaction with many kinds of substrates. We hope that the chemical/physical description of MOCVD, curvature effects, and 3D-S shape, involved during growth phenomena, can help improve material research and present a more general mechanism in order to obtain nanosized materials and devices for future developments. The present discussion can also be applied to devices such as GaInN quantum dots intended for visible laser materials. This information is presented as a challenge to be overcome and not as an end. Nevertheless, considerable information is still needed to build a complete scientific explanation of the wonderful and exciting nanoscience world of dots, wires, and single or multiwalled tubes.

\section{ACKNOWLEDGMENTS}

This work is part of the FILEMON35 project. We acknowledge the financial support of the "Agence Nationale de la Recherche" under Contract No. ANR-05-NANO-016-04.

${ }^{1}$ S. Iijima, Nature (London) 354, 56 (1991).

${ }^{2}$ J. Wang, M. Gudiksen, X. Duan, Y. Cui, and Ch. Lieber, Science 293, 1455 (2001).

${ }^{3}$ M. Gudisksen and Ch. Lieber, J. Am. Chem. Soc. 122, 8801 (2000).

${ }^{4}$ X. Duan and Ch. Lieber, Adv. Mater. (Weinheim, Ger.) 12, 298 (2000); J. Am. Chem. Soc. 122, 188 (2000).

${ }^{5}$ B. Ohlsson, M. Bjork, M. Magnusson, K. Deppert, L. Samuelson, and L. Wallenberg, Appl. Phys. Lett. 79, 3335 (2001).

${ }^{6}$ U. Krishnamachari, M. Borgstrom, B. Ohlsson, N. Panev, L. Samuelson, W. Seifert, M. Larsson, and L. Wallenberg, Appl. Phys. Lett. 85, 2077 (2004).

${ }^{7}$ J. Motohisa, J. Noborisaka, J. Takeda, M. Inari, and T. Fukui, J. Cryst. Growth 272, 180 (2004)

${ }^{8}$ K. Hiruma, M. Yazawa, T. Katsuyama, K. Ogawa, K. Haraguchi, M. Koguchi, and H. Kakibayashi, J. Appl. Phys. 77, 447 (1995).

${ }^{9}$ M. Sacilotti, L. Imhoff, S. Bourgeois, C. Dumas, J. Decobert, P. Baldeck, and I. Colombier, J. Cryst. Growth 261, 253 (2004).

${ }^{10}$ M. Sacilotti, L. Imhoff, C. Dumas, J. C. Vial, P. Baldeck, I. Colombier, and F. Donatini, Jpn. J. Appl. Phys., Part 2 43, L698 (2004).

${ }^{11}$ M. Sacilotti, J. Decobert, H. Sik, G. Post, C. Dumas, P. Viste, and G. Patriarche, J. Cryst. Growth 272, 198 (2004).

${ }^{12}$ R. Wagner and W. Ellis, Appl. Phys. Lett. 4, 89 (1964).

${ }^{13}$ E. Givargizov, J. Cryst. Growth 20, 217 (1973); 31, 20 (1975).

${ }^{14}$ T. Trentler, K. Hickman, S. Goel, A. Viano, P. Gibbsons, and W. Buhro, Science 270, 1791 (1995).

${ }^{15}$ H. Yu, P. Guibbons, K. Kelton, and W. Buhro, J. Am. Chem. Soc. 23 , 9198 (2001)

${ }^{16} \mathrm{H}$. Yu, J. Li, R. Loomis, L. Wang, and W. Buhro, Nature (London) 2, 517 (2003).

${ }^{17} \mathrm{Ph}$. Buffat and J. P. Borel, Phys. Rev. A 13, 2287 (1976)

${ }^{18}$ P. Cheyssac, R. Kofman, G. Mattei, P. Merli, A. Migliori, and A. Stella,
Superlattices Microstruct. 17, 47 (1995).

${ }^{19}$ R. Kofman, P. Cheyssac, Y. Lereah, and A. Stella, Eur. Phys. J. D 9, 441 (1999).

${ }^{20}$ M. Bjork et al., Nano Lett. 2, 87 (2002).

${ }^{21}$ A. Persson, B. Ohlsson, S. Jeppesen, L. Jensen, and L. Samuelson, J. Cryst. Growth 272, 167 (2004).

${ }^{22}$ R. Kofman, P. Cheyssac, A. Aouaj, Y. Lereah, G. Deutscher, T. BenDavid, J. Penisson, and A. Bourret, Surf. Sci. 303, 231 (1994).

${ }^{23} \mathrm{P}$. Cheyssac, R. Kofman, and A. Aouaj, in Physics and Chemistry of Finite Systems: From Clusters to Crystals Vol. 1, NATO Advanced Studies Institute, edited by P. Jena, S. N. Khanna, and B. K. Rao (Kluwer Academic, Dordrecht, 1992), Vol. 1, pp. 387-392.

${ }^{24}$ M. Pinault, V. Pichot, H. Khodja, P. Launois, C. Reynaud, and M. L'Hermite, Nano Lett. 5, 2394 (2005).

${ }^{25}$ S. Lee, N. Wang, and C. Lee, Mater. Sci. Eng., A 286, 16 (2000).

${ }^{26}$ W. Shi, Y. Zheng, N. Wang, C. Lee, and S. Lee, Adv. Mater. (Weinheim, Ger.) 13, 591 (2001).

${ }^{27}$ Czochralski method, Ref. 20 of W. Runyan, Silicon Semiconductor Technology (McGraw-Hill, New York, 1965), pp. 38-49.

${ }^{28}$ A. Dick, K. Deppert, M. Larsson, W. Seifert, L. Wallenberg, and L. Samuelson, J. Cryst. Growth 272, 131 (2004).

${ }^{29}$ J.-J. Métois, Surf. Sci. 241, 279 (1991).

${ }^{30}$ M. Kern, Surf. Sci. 264, 467 (1992).

${ }^{31}$ J. Olives Banos, J. Phys.: Condens. Matter 5, 2081 (1993).

${ }^{32}$ P. G. De Gennes, Rev. Mod. Phys. 57, 827 (1985).

${ }^{33}$ E. Søndergaard, R. Kofman, P. Cheyssac, F. Célestini, T. Ben-David, and Y. Lereah, Surf. Sci. 388, L1115 (1997).

${ }^{34}$ H. Manasevit, J. Cryst. Growth 55, 1 (1981).

${ }^{35}$ J. Nishizawa, H. Sakuraba, and T. Kurabauashi, J. Vac. Sci. Technol. B 14, 136 (1996).

${ }^{36}$ O. Bord, R. Talalev, S. Karpov, and Y. Makarov, Phys. Status Solidi A 176, 297 (1999).

${ }^{37}$ J. Yu, E. Wang, J. Ahn, S. Yoon, Q. Zhang, and M. Yu, J. Appl. Phys. 87, $4022(2000)$

${ }^{38}$ Y. Chen, J. Fitz Gerald, L. Chadderton, and L. Chaffron, J. Metastable Nanocryst. Mater. 2-6, 375 (1999).

${ }^{39}$ Y. Chen, J. Fitz Gerald, L. Chadderton, and L. Chaffron, Appl. Phys. Lett. 74, 2782 (1999).

${ }^{40}$ L. Imhoff, M. Sacilotti, C. Josse Courty, M. Mesnier, M. C. de Lucas, and S. Bourgeois, Phys. Status Solidi C 1, 388 (2004).

${ }^{41}$ Th. Chiaramonte, G. Patriarche, J. Decobert, L. P. Cardoso, and M. Sacilotti, Nanotechnology 16, 2790 (2005).

${ }^{42}$ M. K. K. Nakaema, M. P. F. Godoy, M. J. S. P. Brasil, F. Iikawa, D. Silva, M. Sacilotti, J. Decobert, and G. Patriarche, J. Appl. Phys. 98, 535 (2005).

${ }^{43}$ V. K. Semenchenko, Surface Phenomena in Metals and Alloys (Pergamon, New York, 1961).

${ }^{44}$ P. Cheyssac, M. Geddo, R. Kofman, P. G. Merli, A. Migliori, A. Stella, and P. Tognini, Mater. Sci. Forum 195, 161 (1995).

${ }^{45}$ P. Cheyssac, R. Kofman, P. G. Merli, A. Migliori, and A. Stella, Mater. Res. Soc. Symp. Proc. 332, 108 (1994).

${ }^{46}$ A. Stella, A. Migliori, P. Cheyssac, and R. Kofman, Europhys. Lett. 26, 265 (1994).

${ }^{47}$ R. Kusche, Th. Hippler, M. Schmidt, B. von Issendorff, and H. Haberland, Eur. Phys. J. D 9, 1 (1999).

${ }^{48}$ T. Nguyen, P. S. Ho, T. Kwok, C. Nitta, and S. Yip, Phys. Rev. Lett. 57, 1919 (1986)

${ }^{49}$ J. Q. Broughton and G. H. Gilmer, Phys. Rev. Lett. 56, 2692 (1986).

${ }^{50}$ Y. Lereah, R. Kofman, J. M. Penisson, G. Deutscher, P. Cheyssac, T. Ben David, and A. Bourret, Philos. Mag. B 81, 1801 (2001).

${ }^{51}$ P. Viste et al., J. Cryst. Growth 272, 466 (2004).

${ }^{52}$ D. Chatain, I. Rivollet, and N. Eustathopoulos, J. Chim. Phys. Phys.Chim. Biol. 84, 201 (1987).

${ }^{53}$ D. Chatain, I. Rivollet, and N. Eustathopoulos, Rev. Phys. Appl. 23, 1055 (1988).

${ }^{54}$ M. Volmer and A. Weber, Z. Phys. Chem., Stoechiom. Verwandtschaftsl. 119, 277 (1926).

${ }^{55}$ F. C. Frank and J. H. van der Merve, Proc. Roy. Soc. 198, 216 (1949).

${ }^{56}$ M. Leskela and M. Ritala, Angew. Chem., Int. Ed. 42, 5548 (2003).

${ }^{57}$ T. Nissila, R. Ferrandos, and S. Ying, Adv. Phys. 51, 949 (2002).

${ }^{58}$ L. D. Marks and D. J. Smith, Nature (London) 303, 316 (1983).

${ }^{59}$ J. O. Bovin, R. Wallenberg, and D. J. Smith, Nature (London) 317, 47 (1985). 
${ }^{60}$ Nature (London) 315, 628 (1985).

${ }^{61}$ M. Flüeli, P. A. Buffat, and J. P. Borel, Inst. Phys. Conf. Ser. 8, 311 (1988).

${ }^{62}$ N. Lei, Z. Huang, and S. A. Rice, J. Chem. Phys. 107, 4051 (1997)

${ }^{63}$ A. H. Ayyad and W. Freyland, Surf. Sci. 506, 1 (2002).

${ }^{64}$ Y. Gao, Y. Bando, Z. Liu, and D. Golberg, Appl. Phys. Lett. 83, 2913 (2003).

${ }^{65}$ Y. Gao and Y. Bando, Appl. Phys. Lett. 81, 3966 (2002).
${ }^{66}$ W. J. Huisman, J. F. Peters, M. J. Zwanenburg, S. A. deVries, T. E. Derry, D. Abernathy, and J. F. van der Veen, Nature (London) 390, 379 (1997).

${ }^{67}$ R. W. Cahn, Nature (London) 375, 363 (1995).

${ }^{68}$ B. Q. Wei, Z. J. Zhang, P. M. Ajayan, and G. Ramanath, Carbon 40, 47 (2002).

${ }^{69}$ C. J. Lee and J. Park, Appl. Phys. Lett. 77, 3397 (2000).

${ }^{70}$ B. Q. Wei, Z. J. Zhang, G. Ramanath, and P. M. Ajayan, Appl. Phys. Lett. 77, 2985 (2000). 